\title{
Die
}

\section{Oligochæten von Zürich in}

\section{systematischer u. biologischer Hinsicht \\ von}

\section{K. BRETSGHER}

Mit 4 Figuren im Text

Die vorliegende Arbeit wurde im zoologischen Institut der beiden Hochschulen Zürichs zu Ende geführt und nach Schluss des Wintersemesters 1894-95 seitens der hohen philosophischen Fakultät, II. Sektion, der Universität mit einem Preise bedacht.

$\mathrm{Zu}$ hoher Befriedigung gereicht es mir, den Leiter des zoolog. Institutes, Herrn Prof. $\mathrm{D}^{\mathrm{r}}$ A. LANG, für die vielfach gebotenen Anregungen und die Unterstützung, die er mir zu Teil werden liess, hiemit öffentlich meines innigsten Dankes versichern zu können.

Von einem Litteraturverzeichnis wurde hier Umgang genommen. Wer sich für die Oligochæten speziell interessirt, wird genügende bezügliche Anhaltspunkte finden, in :

VEJDovsky, System und Morphologie der Oligochaeten, Prag 1884.

Michaelsen, Synopsis der Enchytraeiden; Abhandl. des naturwiss. Vereins Hamburg. Hamburg 1889.

Rosa, Revisione dei Lumbricidi. Torino 1893. und namentlich in dem umfassenden neuesten Werke:

BeDDARD, A Monograph of the Order of Oligochaeta. Oxford 1895, dessen System hier zu Grunde gelegt wurde. 


\section{A. Beschreibung der Arten.}

\section{KLASSE : APHANEURA \\ FAMilie : AEOLOSOMATIDA \\ I. Genus: Aeolosoma Ehrenberg.}

Kleine Süsswasserbewohner. Ohne Dissepimente zwischen den einzelnen Segmenten. Kopflappen unterseits bewimpert. Borsten in Bündeln von 1-6, meist haarförmig; in jedem Segment 4 solcher Bündel.

Clitellum nur ventral auf den Segmenten 5-7, Hoden einfach, in 5.; Ovarien, einfach, in 6., ohne differenzirten Samenleiter; Eileiter als ventraler medianer Porus in 6., Spermatheken in 3-4.

Nervensystem nur als Gehirnganglion vorhanden. Am Kopflappen jederseits eine Wimpergrube.

\section{Ae. hemprichii Ehrenberg.}

(Hemprich u. Ehrenberg, Symbolae physicae II, Phytozoa. Decas I. Tafel II. 1828.)

Kopf breiter als die übrigen Segmente. Borsten fast gerade, kürzere und längere in jedem Bündel. Oeldrüsen orangerot. Gehirn hinten durch einen tiefen Einschnitt geteilt. Nephridien im 1. Borstensegment beginnend. Darm von einem Kapillarnetz umfasst.

Fundorte: In Menge fand sich diese Art in einem Graben an der Langmauerstrasse (Zürich IV), dessen Wasser leider bald abgeleitet wurde; spärlicher in der Bucht am Züricherhorn und in den Torftümpeln des Katzensees, zwischen Algen.

\section{Ae. fiedleri nov. spec.}

Länge 1-2 mm. Segmentzahl 12-13. Kopflappen vorn zugespitzt, nicht breiter als die übrigen Segmente. Oeldrüsen farblos, ungleich gross, unregelmässig zerstreut. Haut am Vorder- 
ende drüsig, Tasthaare hier und am Hinterende lang. Borsten zu 3-4 per Bündel, hinten nur zu 2, gleich lang, schwach $\mathcal{S}$ förmig gebogen, mindestens bis zum folgenden Bündel reichend. Gehirn klein, hinten nur wenig ausgebuchtet. Nephridien mit langem verschlungenem Kanal, erstes vor dem 3. Borstenpaar liegend.

Fundort: In den Torftümpeln am Katzensee ziemlich häufig.

Diese Art weicht von Ae. variegatum Vejd. ab in der Kopfbildung und Borstenlänge, von Ae. tenebrarum Vejd. in der Grösse und Borstenform, von Ae.niveum Leydig in der Borstenform und der Zahl der Nephridien, so dass die Abtrennung als eigene Spezies, die ich dem leider allzu früh verstorbenen, talentvollen Zoologen, Dr Karl FiEdLER von Zürich, widmen möchte, berechtigt erscheinen dürfte.

\section{KLASSE : MICRODRILI}

FAMILIE : PHREORYCTIDE

Wasser- oder Erdbewohner von oft sehr bedeutender Länge. 4 Borstenreihen in Bündeln von 1-2, sigmoid. Hoden in 10. und 11., Ovarien in 12., 13. oder nur 13. 2 Paar getrennt sich öffnender Samenleiter. Spermatheken vor den Hoden liegend.

\section{Genus : Phreoryctes Hoffmeister.}

Borsten in 4 Reihen, einzeln und paarig. Clitellum 11-14. Prostomium durch eine Querfurche geteilt. Hoden in 10. und 11.; Ovarien in 12. und 13., Samenleiter in 11. und 12., Eileiter in den Intersegmentalfurchen 12-13 und 13-14 austretend. 2-3 Paar Spermatheken in 7., 8 (9).

1. Ph. menkeanus Hoffmeister.

(Hoffmeister, Die bis jetzt bekannten Arten aus der Familie der Regenwürmer; 184气.)

Jänge bis $60 \mathrm{~cm}$.; Durchmesser 1-2 mm.; über $500 \mathrm{Seg}$ mente. Ventrale Borsten länger als die dorsalen, immer einzeln. 
Ein Exemplar im zoologischen Museum stammt vom Zürichberg (Fluntern).

2. Ph. filiformis Claparède.

(Claparède, Recherches anatomiques sur les Oligochètes. Mémoires de la Société de physique et d'histoire naturelle. Genève 1862. 16, II, p. 217-291.)

Länge 120-140 mm., Durchmesser 1-2 mm., Borsten einzeln, dorsale länger als die ventralen. 260 Segmente.

Fundorte: In feuchter Erde an einem Wassergraben in Zürich IV (fallender Brunnenhof); an Algen in der Rhône (ClaPARÈDE).

\section{FAMILIE : LUMBRICULID无}

Wasserbewohner von mittlerer Grösse. Borsten paarig und $\mathcal{f}$-förmig, oft mit gabeligem freiem Ende. Rückengefäss und Seitengefässe mit blinden kontraktilen Anhängen (Stylodrilus ausgenommen). 2 Paar Samenleiter, die jederseits in eine gemeinsame, vor der Eileiteröffnung gelegene Drüse münden. Penisborsten fehlen.

\section{Genus : Lumbriculus Grube.}

Distales Ende der Borsten gegabelt. Von 8. an in jedem Segment ein Paar blind endigender, verzweigter Seitenäste des Rückengefässes und ein nicht kontraktiler Perivisceralast, der dieses mit dem Bauchgefäss verbindet. Hoden in 8., Spermatheken in 10., 11. und 12., Eileiter in 10. und 11., Eiweissdrüse in 9.

\section{L. variegatus $\mathrm{O}$. F. Müller.}

(0. F. Müller, De vermium terrestrium et fluviatilium... historia, 1773.)

Länge $80 \mathrm{~mm}$. Segmente 200 ; vorn dunkelgrün oder dunkelbraun; in jedem der hintern Segmente 6-8 blinde Aeste des Rückengefässes.

Fundorte: Häufig in der Limmat, im Zürich- und Katzensee, in kleineren Bächen und Tümpeln. 
IV. Genus: Stylodrilus Claparède.

(Claparède, Recherches... cit.)

Borsten gegabelt. Clitellum in 11-12., Spermatheken 1 Paar in 9., 1 Paar nicht kontraktiler Penes in 10.; Samenleiterdrüsen birnförmig mit langem Kanal.

1. St. gabretae Vejdovsky.

(Vejdovsky, Revisio Oligochaetorum Bohemiae. Sitzungsberichte d. k. böhm. Gesellschaft d. Wissenschaften. Prag 1883.)

Länge ca. $40 \mathrm{~mm}$. Segmente 80. Prostomium konisch. Rückengefäss mit Erweiterungen; Seitenherzen in 6. und 7.; Penis fast so lang als der Durchmesser des Körpers.

Fundort: Limmat, beim Ausflusse des Zürichsees, ziemlich häufig.

\section{Genus: Claparedilla Vejdovsky.}

Borsten nicht gegabelt; 2 Paar kontraktiler Perivisceralschlingen mit blinden Anhängen in jedem der mittleren und hinteren Segmente. Spermatheken in 9.

\section{C. meridionalis Vejdovsky.}

(Vejdovsky, Revisio... cit.)

Prostomium so lang als das Mundsegment; vordere Perivisceralschlinge in jedem Segment mit Chloragogenzellen bedeckt, beim Eintritt in's Bauchgefäss erweitert und 4-5 blinde Aeste abgebend; hintere Schlinge mit Reihen von Aesten in fiedriger Anordnung.

Fundort: Genfersee; aus einer Sendung, die ich der gütigen Vermittlung des Herrn Prof. Fonel in Morges und der grossen Gefälligkeit von Herrn Prof. Blanc in Lausanne verdanke. Claparède gibt als Fundort an: «le lit du Rhône à la Coulouvrenière et dans le lit de la Seime. » 


\section{FAMILIE : TUBIFICID $\mathrm{E}$}

Dünne, langgestreckte Wasserbewohner. 3 Borstenformen: haarförmige, kammförmige und gabelige; die ersten 2 nur dorsal. Bauch- und Rückengefäss in jedem Segment durch periviscerale Seitenschleifen verbunden. Hoden in 10., Ovarien in 11., Samenleiter immer mit Drüse, in 11. sich öffnend. Mündung der Eileiter in 11-12. Spermatheken, 1 Paar, in 10. Oft Penisborsten.

VI. Genus : Tubifex Lamarck.

Dorsale Borstenbündel mit Haar-, Kamm- und Hakenborsten, ventrale nur mit hakigen. Seitenherzen in 8. Ohne chitinösen Penis.

\section{T. rivulorum Lamarck.}

(0. F. Müller, De vermium terrestrium historia, 1774. Lumbricus tubifex.)

Gehirn vorn konkav mit deutlichen Seitenlappen, hinten 3-spaltig mit kleinem mittlerem und 2 grösseren seitlichen Einschnitten. Dorsale Borsten haar-, kamm- und hakenförmig.

Fundorte: Gemein im Schlamme der Limmat, in den Weiern im Dratzug (Zürich V) und Fluntern; weniger zahlreich im Zürich- und Katzensee, in den Tümpeln im Hard (zwischen Zürich und Altstetten), in Bächen (Letzibach).

\section{Genus : Limnodrilus Claparède.}

Süsswasseroligochæten mit einer einzigen Borstenform (Hakenborsten). Seitenherzen in 8. oder 8. und 9. Die Perivisceralschlingen der hintern Segmente geben Aeste an die Leibeswand ab. Penis mit chitinöser Wandung. Prostatadrüsen vorhanden.

\section{L. claparedianus Ratzel.}

(Ratzel, Beiträge zur anatomischen und systematischen Kenntnis der Oligochaeten. Zeitschrift f. wiss. Zoologie, 18, 1868.)

Länge ca. $50 \mathrm{~mm}$. Segmente 90-200. Gehirn fast viereckig, 
DIE OLIGOCHETEN VON ZURICH.

mit viereckigem hinterem Einschnitt, vorderer Seitenlappen in 2 geteilt, der eine nach vorn, der andere nach hinten gerichtet. Pharynx bis in's 5. Segment reichend. Chitinöser Penis 8-10 mal länger als breit.

Fundorte: Limmat beim Ausfluss aus dem Zürichsee; Lettenkanal.

2. L. hoffmeisteri Claparède.

(Claparède, l. c.)

Länge ca. $30 \mathrm{~mm}$. Segmente bis gegen 100 . Hirn viereckig mit schwacher hinterer Ausbuchtung. Pharynx in's 3. Segment reichend. Nephridien mit bläschenartigen Zellen. Penis 6-7 mal so lang als breit.

Fundorte: Im Weier an der Gloriastrasse Zürich V, im Lettenkanal, in der Limmat, in den Weiern im Dratzug, nicht gerade zahlreich vorkommend.

3. L. udekemianus Claparède. (Claparède, 1. c.)

länge 20.60 mm. 100 Segmente. Gehirn mit hinterem mittlerem und seitlichen Lappen, mit paarigem vorderem $\mathrm{u}$. vorderen seitlichen Lappen. Pharynx bis 5. reichend. Penisscheide 3 mal länger als breit.

Fundorte: Wie vorige Art, ferner in der Umgebung von Dunghaufen (Hittnau) sehr zahlreich.

VIII. Genus: Psammoryctes Vejdovsky.

Borsten haar-, haken-, kamm- und fächerförmig. Samenleiterdrüse mit einer Samenblase. Prostata wie in Tubifex. Spermatheken gemeinsam mit einer muskulösen Tasche sich öffnend, die eine lange Geschlechtsborste enthält ; dem Behälter sind 2 oder 4 Drüsen angefügt.

\section{P. barbatus Grube.}

(Grube, Ein A usflug nach Triest und Quirnero, 1861: Sænuris barbata.)

Länge $30-40 \mathrm{~mm}$. Segmente 40-90. Prostomium so lang 
als das Mundsegment. Hakenborsten mit gleich grossen Zinken oder der eine länger als der andere; erstere Form vom 11. Segment an nach hinten dorsal und ventral, die andere in den ventralen vordern Bündeln; ohne Borsten in 11; Fächerborsten in $2-10$.

Fundorte: Zürichsee im Uferschlamm und in der Tiefe, im Schanzengraben, in der Limmat und im Lettenkanal häufig.

IX. Genus : Embolocephalus Randolph.

Dorsal nur Haar- oder auch Hakenborsten. Vorderste Segmente zurückziehbar. Nicht retraktile Sinnesorgane in Querreihen. Aufenthalt in selbsthergestellten Röhren. Samenleiterdrüsen wie in Tubifex.

1. E. velutinus Grube.

(Grube, Untersuchungen über die physikalische Beschaffenheit und die Flora und

Fauna der Schweizerseen. Jahresberichte der Schlesischen Gesellsch. für vaterländ. Kultur, 1878. Sænuris velutinus.)

Länge 30-50 mm. Segmente 50-70. 2 Reihen von Sinnespapillen in jedem Segment. Rückenborsten haarförmig, 2-4 per Bündel. Ventrale Borsten zu 2, am Ende nicht immer deutlich gegabelt. Gehirn vorn konkav mit 2 hinteren Lappen. Clitellum in 10-12; in 10. ein Paar grosser Drüsen mit den ventralen Borsten verbunden.

Fundort: Im Tiefseeschlamm des Zürichsees sehr häufig.

2. E. plicatus Randolph.

(Randolph, Ein Beitrag zur Kenntnis der Tubificiden. Vierteljahrsschrift der Naturforsch. Gesellsch. Zürich, 37, 18.2.)

Länge ca. $40 \mathrm{~mm}$. Segmente 50. 2 oder mehr Reihen von Sinnespapilleı um jedes Segment. Dorsal in jedem Bündel gewöhnlich 3 Paar haarförmige und 3 kurz gespaltene Borsten, ventral $2-5$ stark $\mathcal{f}$-förmige. Clitellum in 10-12.

Fundorte : Zürichsee im Tiefseeschlamm neben voriger, recht vereinzelt; in der Limmat (Schipfe, Lettenkanal) ausschliesslich und häufig. 


\section{FAMILIE : NAIDOMORPHA}

Kleine Wasserbewohner. Borsten meist in 4 Bündeln in jedem Segment, $\mathcal{f}$-förmig geschwungen, hakig und haarförmig. Geschlechtliche Vermehrung in bestimmten Intervallen; daneben ungeschlechtliche durch Teilung. Geschlechtsorgane weit vorn gelegen, schon im 5. Segment beginnend.

X. Genus: NaIs O. F. Müller.

Dorsale Borsten in 6 . beginnend, nur haarförmige, oder neben diesen auch hakige oder eng gegabelte. Augen vorhanden.

1. N. barbata O. F. Müller.

(0. F. Müller, De vermium... historia 1774.)

Dorsale Bündel nur mit haarförmigen Borsten von verschiedener Grösse, zu 4-8. Augen vorhanden.

Fundorte: Im Zürichsee, in der Limmat und dem Lettenkanal, im Weier in Fluntern an Wasserpflanzen.

2. N. elinguis O. F. Müller. (O. F. Müller, I. c.)

Dorsal haarförmige und gegabelte Borsten, zu 1-3 per Bündel. Augen vorhanden.

Fundorte: Ueberall, in allen unsern Gewässern an Wasserpflanzen, an Steinen, auf dem Schlamm sehr gemein.

3. N. lacustris Linné.

(Linnæus, Systema naturae 1767 : Nereis lacustris.)

Länge 15 mm.; 25 Segmente. Prostomium sehr verlängert. Dorsal nur Haarborsten. Mit Augen.

Fundorte: Im Zürichsee an Wasserpflanzen überall sehr häufig; ebenso in der Limmat, in den Hardtümpeln und im Katzensee.

4. N. serpentina O. F. Müller.

(O. F. Müller, 1. c.)

Dorsale Borsten kurz, dünn und gegabelt; ventrale der vor- 
dern 4 Bündel mit mittlerem Nodulus, an den folgenden ist er näher dem proximalen Ende. In den Segmenten 2-5. drei oder 4 dunkle Pigmentstreifen.

Fundorte: Zürichsee, Wassertümpel im Hard gemein neben den vorgenannten 2 Arten; im Katzensee scheint er zu fehlen.

5. N. appendiculata d'Udekem.

(D'Udekem, Nouvelle classification des Annèlides sétigères abranches. Bulletin de l'Académie royale de Belgique, 22, 18ว̈5.)

Dorsal nur Haarborsten, die des 6. Segmentes länger als die übrigen. Eine Reihe von Sinnespapillen auf jedem Segment.

Fundorte: Hardtümpel, Lettenkanal, Torftümpel am Katzensee häufig. Aus dem Zürichsee verzeichnete ich keinen Fund.

XI. Genus : Pristina Ehrenberg.

Dorsale Borsten im Segment 2 beginnend. Nur Haar- oder daneben auch Nadelborsten; letztere können am Ende auch gegabelt sein. Augen fehlen. Septaldrüsen deutlich.

1. P. longiseta Ehrenberg.

(Hemprich und Ehrenberg, Symbolx physicæ cit.)

Länge 8 mm. 20 Segmente. Die dorsalen Borsten in 3. viel länger als die übrigen. Prostomium rüsselartig verlängert. Drüsenventrikel in 8. 5 Schleifen vom Rücken- zum Bauchgefäss tretend. Erstes Nephridienpaar in 10.

Fundorte : Einzelne Torftümpel in der Umgebung des Katzensees, zwischen Algen; andere Fundstellen konnten nicht konstatirt werden.

XII. Genus: Номоснжта nov. gen.

Alle Borsten doppelhakig, dorsal schon in 2. beginnend, zu 3-6, ventral zu 3-5 per Bündel.

1. H. naidina nov. spec.

Rüssel fehlend; Kopflappen spitzig, etwa 3 mal länger als ein Borstensegment. Augen vorhanden oder fehlend. Kopfporus 
scheint vorhanden. Borsten wie angegeben; die Cephalisation ist dadurch ausgesprochen, dass die dorsalen Borsten der Segmente $2-5$ schlanker sind als die übrigen, zu $5-6$; von 6 . an entsprechen sie den ventralen und bilden zu 3-5 ein Bündel. Bei jenen liegt der Nodulus in der Mitte und ist der obere Haken länger als der untere; der Nodulus der übrigen Borsten liegt im distalen Drittel.

Die vordersten Borsten stehen ganz nahe der Mundspalte, so dass das erste Segment sehr verkürzt erscheint.

Das Vorderende ist mit gelbbräunlichem Pigment durchsetzt (Pharyngealregion) und trägt wie das Hinterende lange Tasthaare; am übrigen Körper stehen diese vereinzelt. 20 Segmente bei $8 \mathrm{~mm}$. Länge; mit Knospungszone.

In jedem Segment geht eine Schleife vom Bauchgefäss zum Darm ; die 5 vordern zeigen die Verbindungen zwischen Rückenund Ventralgefäss in ein Kapillarnetz aufgelöst.

In 8. eine deutliche Erweiterung des Darmes (Drüsendivertikel).

Gehirn schmal, hinten tief, vorn wenig eingeschnitten.

Fundort: Limmat an Wasserpflanzen, am Ausfluss des Zürichsees in wenigen Exemplaren.

XIII. Genus: Macrocheta nov. gen.

1. $M$. intermedia nov. spec.

Länge 5 mm. Segmentzahl 15-20. Augen fehlen. Haut drüsig. Dorsale Borsten in 6 beginnend, je mit 4-7-9 Haarborsten von verschiedener Länge; die längsten doppelt so lang als der Querdurchmesser des Tieres, nicht gesägt. Ventrale Borsten in 2-5. schlank, zu 2-4, Nodulus in der Mitte; von 5 . an nur je 1 Borste, Nodulus mehr nach aussen; oberer Haken länger als der untere. Gehirn hinten tiefer eingeschnitten als vorn. Darm zwischen den 3-7. Rückenborsten erweitert, das Lumen des Hautmuskelschlauches fast ausfüllend (ähnlich Chaetogaster).

Haut drüsig.

Rev. SUISSE de Zool., T. III. 1896. 
Diese Art ist nahe verwandt mit Ripistes Dujardin sowohl als mit Bohemilla Vejdovsky, weicht jedoch in den Borstenverhältnissen wesentlich von beiden ab.

$\begin{array}{cccc} & \text { Ripistes. } & \text { Bohemilla. } & \text { Macrochæta. } \\ \text { Augen : } & \text { vorhanden. } & \text { vorhanden. } & \text { fehlend. } \\ \text { ALe Bonsten : } & \text { nicht gesägt } & \text { gesägt } & \text { nicht gesägt } \\ & \text { in } 6 . & \text { in } \check{5} . & \text { in } 6 \text {. beginnend } \\ \text { erste } 3 \text { Büindel lang. } & \text { in allen lang. } & \text { in allen lang. }\end{array}$

Bauchborsten : fehlen in 4. u. כ., vorhanden in 4. u. 5., können in 4. fehlen

Fundort: In der Limmat beim Bauschänzli, in Schlamm.

XIV. Genus: Dero Oken.

Rückenborsten haar- und kurznadelförmig, in 6 . beginnend. Am Hinterende des Körpers Kiemenanhänge. Augen fehlen. Röhren bewohnend.

\section{D. furcata Oken.}

(Oken, Lehrbuch der Naturgeschichte, III, 1815.)

Segmente 35. Der Körper endigt in 2 lange Taster. 5 Paar kontraktiler Herzen. (6 Kiemenzapfen.)

Fundort: Zahlreich in einem Torftümpel am Katzensee.

2. D. perrieri Bousf.

(Bousfield, On the Annelids of the Genus Dero. Report of the British Association, 188..)

Segmente 35. Kiemenfläche ganzrandig. 3-5 Paar Seitenherzen. (4 Kiemenanhänge.)

Fundort: In der Limmat beim Bauschänzli.

$$
\text { 3. D. digitata O. F. Müller. }
$$

(0. F. Müller, Von den Würmern des süssen und salzigen Wassers, 1771 : Das blinde Blumentier und De vermium, 1773: Nais digitata.)

6 deutliche Kiemenanhänge (vielleicht ist diese Art identisch mit D. limosa Leidy).

Fundort: Am Zürichhorn zwischen Algen, zahlreich, aber nur während kurzer Zeit. 
XV. Genus: Chætogaster von Bær.

Nur ventrale Borsten vorhanden; diese ausschliesslich hakig; in 2. auftretend; in 3-5. fehlend. 1 Paar Seitenschleifen vom Rücken- zum Bauchgefäss.

1. Ch. limnaei von Bær.

(Von Bær, Beiträge zur Kenntnis der niedern Tiere, Nova acta Acad. Leop. Carol. XIII, 2.)

Länge $2 \mathrm{~mm}$. Oesophagus sehr reduzirt.

Fundorte: Im Katzensee, in den Atemhöhlen von Wasserschnecken (Limnaeus) lebend; aus der Bucht am Zürichhorn fand sich einer frei lebend zwischen Algen.

2. Ch. diaphanus Gruithuisen.

(Gruithuisen, Ueber die Nais diaphanus und diastrophus. Nova acta Ac. Leop. Carol. XIV, 1.)

Länge $15 \mathrm{~mm}$. Oesophagus deutlich, aber kürzer als der Pharynx. Vetrovermis hyalinus Imhof (Zool. Anz. 9, Ein neues Mitglied d. Tiefseefauna d. Süsswasserbecken) dürfte mit dieser Art identisch sein.

Fundorte : Häufig an Wasserpflanzen im Zürichsee und in der Limmat, im Schanzengraben, in den Torftümpeln des Katzensees.

3. Ch. cristallinus Vejdovsky.

(Vejdovsky, Sitzber. cit.)

Länge 2-3 mm. Rücken- und Bauchgefäss nicht in den Pharynx reichend; Oesophagus so lang als dieser.

Fundort: In Tümpeln am Katzensee, namentlich an Myriophyllum.

4. Ch. diastrophus Gruithuisen.

(Gruithuisen, Nova acta...cit.)

Länge 2-5 mm. Blutgefässe normal. Oesophagus so lang als der Pharynx.

Fundorte: Sehr häufig im Katzen-, Zürichsee und in der Limmat. 


\section{Ch. langi nov. spec. ${ }^{1}$}

Grösse 1-2 mm. Vorderende stumpf, abgerundet. Haut durchsichtig. Borsten nur zu 4, Form wie bei voriger Art.

Oesophagus deutlich, lang. Gehirn undeutlich begrenzt, Bauchmark namentlich unter dem Oesophagus mit stark vor-

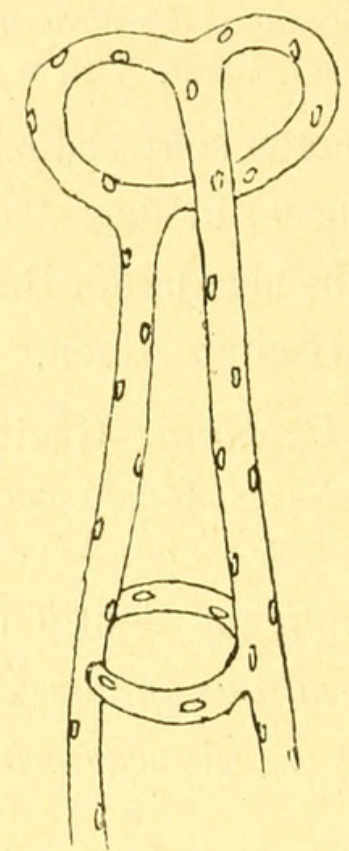

Fig. 1. Chaetogaster langi, zerstrente Zellen auf den Blutgefässen.

springenden, fast nierenförmigen Anschwellungen. Gefässsystem gleich dem von diastrophus, mit weit zerstreuten hyalinen Zellen in zierlicher Weise besetzt. (Bei diastrophus liegen sie so nahe beisammen, dass die Oberfläche in kontrahirtem $\mathrm{Zu}$ stande runzelig wird.)

Die Borstenverhältnisse, die Form der Bauchganglien, der Zellbelag der Blutbahnen unterscheiden diese Art leicht von den übrigen Spezies. Des letzteren Umstandes wegen könnte sie für ein Jugendstadium von diaphanus gehalten werden. Dagegen konstatirte ich an einem Exemplar unmittelbar hinter

${ }^{1}$ Ich benenne diese schöne Art nach meinem hochverehrten Lehrer, Herrn Prof. Dr A. Lang in Zürich. 
dem Oesophagus eine deutliche Gürtelbildung ; dieses Clitellum bestand aus einer Ringzone von einfachen Drüsenzellen.

Fundort: Torftümpel am Katzensee, an Myriophyllum, neben diastrophus und cristallinus.

\section{FAMILIE : ENCHYTRAIDA}

Borsten kurz, gerade oder gebogen, am Ende nicht gegabelt (in Anachaeta fehlend). Bisweilen 1 Paar Kalkdrüsen vorhanden. Rückengefäss vorn immer vorhanden, oft auch im Herzkörper. Hoden in 11., männliche Geschlechtsöffnung in 12.; reduzirte Samenleiterdrüse; Ovidukte als Pori vorhanden. 1 (selten 2) Paar Spermatheken in 5., mit oder ohne Divertikel, meist in den Darm mündend; oft mit Rückenporen.

\section{Genus: Pachydrilus Claparède.}

Borsten $\mathcal{f}$-förmig. Kopfporus dorsal zwischen Prostomium und Mundsegment, ohne Rückenporen. Gehirn hinten eingeschnitten. Blut gelb oder rot; Rückengefäss hinter dem Clitellum entspringend und ohne Herzkörper. Ohne Speicheldrüsen. Hoden mehrfach; Samenleiter lang. Kopulationsdrüsen oft vorhanden.

\section{P. pagenstecheri Ratzel.}

(Ratzel, Beiträge zur anatomischen und systematischen Kenntnis der Oligochaeten. Zeitschrift für wissenschaftl. Zoologie 1868.)

Länge 15-20 mm. Segmente 55-60. Dorsal 3-5, ventral 7-10 Borsten per Bündel. Gehirn vorn konkav. Spermatheken mit deutlichem Gang, der zweimal länger als der Behälter und mit Drüsen besetzt ist.

Fundort: Weier an der Gloriastrasse in Zürich V, zahlreich zwischen Algen.

2. P. maculatus nov. spec.

Länge $5 \mathrm{~mm}$. Segmentzahl 30. Eigentümlich für dieses Tier sind eine Anzahl ockergelber, zerstreuter, grosser Hautdrüsen 
von langgestreckter Form im Kopf- und Analsegment. - Die hellen, farblosen Hautdrüsen sind in die Länge gezogen und liegen in den Segmenten 8-11 in je 2 unregelmässigen Reihen.

Haut dick, derb, durchsichtig. Borsten zu 3-4 per Bündel, $\mathcal{S}$-förmig. Gehirn länger als breit, hinten eingebuchtet. Nephridien mit grossem Postseptale und dünnem Endgang. Samentrichter (Fig. 2) 2 mal länger als breit, mit deutlichem Kragen,

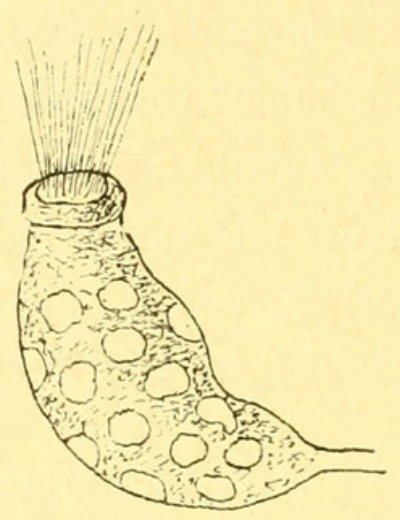

Fig. 2. Pachydrilus maculatus, Samentrichter.

mit hellen, grossen Drüsen besetzt. Samenfäden braun. Samenleiter sehr lang, vielfach gewunden.

Receptacula mit kurzem Gang (Fig. 3), die grosse innere Er-

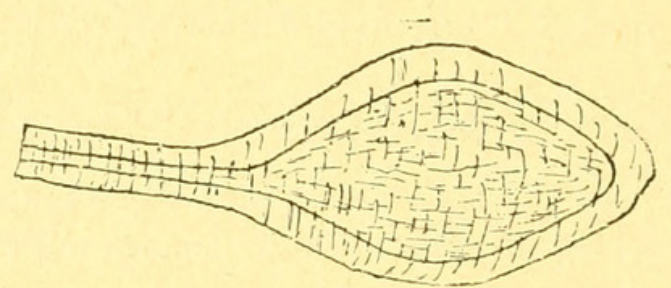

Fig. 3. Pachydrilus maculatus, Receptakulum.

weiterung mit dem Darm zusammenhängend. Geschlechtsreif im September.

Fundort: Hittnau, in der durchtränkten Erde in der Umgebung eines Düngerstockes.

XVII. Genus : Enchytræus Henle.

Borsten eines Bündels gleich lang, gerade, nur am innern Ende umgebogen. Kopfporus zwischen Kopf- und Mundsegment. 
DIE OLIGOCHETEN VON ZURICH.

Oesophagus allmälig in den Darm übergehend. Rückengefäss hinter dem Gürtel entspringend.

1. E. buchholzii Vejdovsky.

(Vejdovsky, Zur Anatomie und Systematik der Enchytraeiden. Sitzungsberichte der k. böhm. Ges. d. Wiss. 1877.)

Länge $10 \mathrm{~mm}$. Segmentzahl 28. Borsten zu 2-3. Gehirn hinten leicht eingebuchtet. Nephridien mit dünnem Anteseptale, geradem Lumen, ohne differenzirten Kanal. Samentaschen mit einem Kanal von der Länge des Behälters und einer Drüsengruppe am Porus.

Fundorte: Weier in Zürich V, an Algen; in Gartenerde.

XVIII. Genus : FrIDERICIA Michaelsen.

Borsten gerade; innere eines Bündels, wenn mehr als 2, kürzer. Kopfporus zwischen Kopf- und Mundsegment; Rückenporen vorhanden. Speicheldrüsen vorhanden. Rückengefäss hinter dem Clitellum entspringend. Blut farblos. Spermatheken in der Regel mit Anhängen. Kopulationsorgane meist vorhanden.

1. F. leydigii Vejdovsky.

(Vejdovsky, Sitzungsber. Prag 1877, cit.)

Länge bis $20 \mathrm{~mm}$. Segmentzahl 40-50. Borsten zu 2-4 per Bündel. Anteseptale gross, braun, mit gewundenem Gang. Spermatheken mit 2 grossen Seitentaschen, ohne Drüsen am Grunde.

Fundort: Modererde am Katzensee.

2. F. perrieri Vejdovsky.

(Vejdovsky, Sitzungsber. Prag 1877, cit.)

Länge bis $20 \mathrm{~mm}$. Segmentzahl 55-60. Borsten zu 4-6, in den ventralen Bündeln mehr. Anteseptale lang und schmal, mit gewundenem Gang. Spermatheken mit 2 Divertikeln, die am blinden Ende erweitert sind. Speicheldrüsen mit 2 knäuelartigen Aesten. 
Fundorte: Gartenerde (Zürich), in Wiesen im Tierfehd (Glarus).

3. F. galba Hoffmeister.

(Hoffmeister, Beitrag zur Kenntnis deutscher Landanneliden. Archiv für Naturgeschichte 1843.)

Länge $20 \mathrm{~mm}$. Segmente 50-70. Borsten zu 4-6; Anteseptale gross und breit, mit gewundenem Gang. Spermatheken mit 4 gestielten Taschen.

Fundorte : In Baummoder und Gartenerde in Zürich, im Tierfehd (Glarus), Cresta (Avers) $1950 \mathrm{~m}$.

4. F. helvetica nov. spec.

Länge 5-6 mm. Segmente 30-40. Farbe milchweiss. Die beiden vordersten Segmente mit grossen glänzenden Drüsen besetzt. Kopfporus gross. Borsten zu 2-4. Lymphzellen in kompakten Ballen beisammen, durchscheinend, mit schwarzen

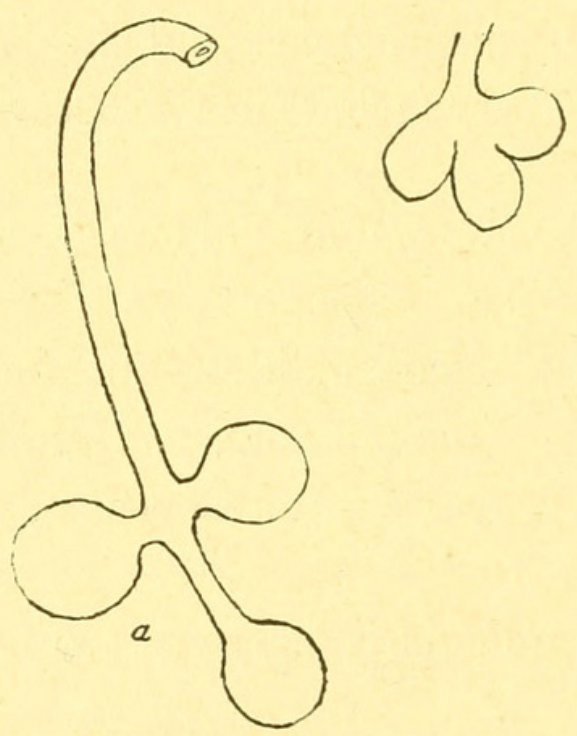

Fig. 4. Fridericia helvetica, $a$ ausgewachsene, $b$ noch unentwickelte Samentasche.

Punkten bestreut; daneben noch kleine hyaline. Gehirn hinten gerade abgestutzt, vorn in der Mitte vorgewölbt, länger als breit. Speicheldrüsen lange, unverzweigte, in Segment 4 gelegene knäuelige Schläuche. Anteseptale halb so gross als das Postseptale. 
Samentrichter mit Kragen, 3 mal länger als breit, Oberfläche drüsig runzlig.

Samentaschen (Fig. 4) lange enge Schläuche mit 2 gestielten kleinen seitlichen Taschen und 1 solchen am Ende des Kanals.

Fundort: Im Mulm alter Weidenstöcke an der Limmat neben Henlea leptodera.

XIX. Genus: Henlea Michaelsen.

Borsten verschieden in Form und Anordnung. Kopfporus klein, zwischen Prostomium und Mundsegment. Rückenporen fehlen. Speiseröhre eng und scharf vom Magendarm abgesetzt. Rückengefäss vor dem Gürtel entspringend. Blut farblos. Lymphkörper in der Regel gross, scheibenförmig. Gehirn hinten ausgeschnitten. Der Ausführungsgang der Segmentalorgane entspringt in der Nähe des dissepimentalen Halses aus dem Postseptale. Samentaschen meist einfach, mit umgekehrt birnförmiger Anschwellung des Samenraumes.

1. H. ventriculosa D'Udekem.

(D’Udekem, I. c.)

Länge 15-20 mm. Segmente 60. Borsten gerade oder leicht geschwungen. 4 Oesophagealdrüsen.

Fundorte: Wassergraben im Rötel, Zürich IV, Weier in Zürich V (Fluntern).

2. H. leptodera Vejdovsky.

(Vejdovsky, Monographie der Enchytraeiden. Prag 1879.)

Länge $20 \mathrm{~mm}$. Segmentzahl 60. Borsten gerade, zu 4-7. 2 Oesophagealdrüsen, mit einfachem Lumen.

Fundorte: In Mulm an den Weiden längs der Limmat (unterhalb Zürich), in Erde, in Baummoder am Katzensee, in Blumentöpfen.

XX. Genus : Anacheta Vejdovsky.

Borsten fehlend. Borstensäcke zu grossen, in die Leibeshöhle hineinragenden Drüsen umgebildet. Kopfporus gross, an der 
Spitze des Kopflappens gelegen. Rückenporen fehlend. Rückengefäss vor den Gürtelsegmenten entspringend. Blut farblos. Gehirı hinten konvex. Eine unpaare Speicheldrüse mündet hinter dem Schlundkopf in den Darm ein. Die Samenkanäle zeigen Neigung zu spiraliger Aufrollung. Samentaschen nicht mit dem Darm verwachsen.

\section{A. bohemica Vejdovsky.}

(Vejdovsky, Vorläufige Mitteilungen über fortgesetzte Oligochaetenstudien. Zoolog. Anzeiger 1879.)

Nur 2 Reihen von Borstendrüsen, deren Lage den Rückenborsten entspricht.

Fundort: In Wiesen bei Zürich IV, in wenigen Exemplaren.

\section{FAMILIE : LUMBRICID A}

Erd- (selten Wasser-) bewohner, meist von geringer Grösse ; 8 Borsten in jedem Segment. Männliche Geschlechtsöffnung in 12., 13. oder meistens in 15. Gürtel sattelförmig, hinter jener gelegen. Rückenporen vorhanden. Kropf meist einfach, am Ende des Oesophagus gelegen. Nephridien paarig und alle einander ähnlich. Ohne Samenleiterdrüse oder Penisborsten. Tubercula pubertatis fast immer vorhanden.

XXI. Genus : Allurus Eisen.

Männliche Geschlechtsöffnung in 13. Gürtel in 21-26. Borsten paarig; die des Gürtels von besonderer Form.

1. A. tetraedrus Savigny.

(Savigny, Analyse d'un mémoire sur les Lombrics par Cuvier.

Mém. Acad. Roy. Inslit. Fr. 1826.)

Länge 35-50 mm. Segmente 70-90. Guirtel 22., 23-26., 27. Tubercula pubertatis 23-25., 26. Rückenporen in 4-5. beginnend. Spermatheken in 9. und 10., ausser der 4. Borste mündend. 
DIE OLIGOCHETEN VON ZURICH.

Fundorte: In allen unsern Wassergräben, Bächen, Ufern der Flüsse und Seen, sehr gemein.

XXII. Genus : Allolobophora Eisen.

Prostomium den Mundlappen unvollständig teilend. Borsten paarig oder abstehend. Männliche Geschlechtsöffnung in 15. Ohne mediane Samenblase.

\section{A. fretida Savigny.}

(Savigny, l. c.)

Farbe : Jedes Segment dorsal mit rötlicher Querbinde, intersegmental gelb.

Länge 80-90 mm. Durchmesser 3-4 mm. Segmente 100 . Prostomium $1 / 2{ }^{1}$, Fortsatz breit. Borsten eng paarig. Clitellum 24., 25., 26-32. Tubercula pubertatis 28,, 29., 30 (31). Rand der $\sigma^{\Upsilon}$ Geschlechtsöffnung angeschwollen. Erster Rückenporus in 4-5.; beim Abtöten scheiden die Tiere durch diese Poren reichlich einen gelben Schleim aus.

Fundorte: In Kompost- und Düngerhaufen gemein ; auf der Halbinsel Hurden (Zürichsee); auf der Frutt wurde sie von Herrn Prof. Dr StoLl gefunden.

\section{A. rosea Sav.}

(Savigny, loc. cit.)

Farbe blass fleischrot, Gürtel rötlich gelb.

Form cylindrisch; die in Alkohol konservirten Tiere zeigen ein sehr verbreitertes Clitellum (Formol bewirkt diese Veränderung nicht).

Länge 60-70 mm. Segmente 125-150. Prostomium $1 / 2$. Clitellum 25., 26-32. Tub. pubertatis 29., 30., 31. Borsten eng paarig. $\sigma^{7}$ Geschlechtsöffnung deutlich, angeschwollen. 1 Rückenporus in 4-5., an in Alkohol konservirten Exemplaren sehr deutlich auch auf dem Gürtel.

${ }^{1}$ Der Prostomiumfortsatz geht bis in die Mitte des Mundsegmentes. 
Fundorte: In der ganzen Umgebung von Zürich, im Tierfehd (Glarus), in Thusis, Cresta (Avers) in Wiesen zahlreich.

3. A. veneta Rosa.

(Rosa, Note sui lombrici del Veneto; Atti del R. Istituto veneto di Scienze, Venezia 1886.)

Länge $70 \mathrm{~mm}$. Durchmesser $5 \mathrm{~mm}$. Segmentzahl 90-110. Prostomium $2 /{ }_{3}$. Gürtel 26., 27-32., 33. Tub. pub. 30, 31. $\checkmark$ Oeffnung seitlich gelegen.

Erster Rückenporus in 5-6.

A. veneta var. typica Rosa.

Borsten fast gleich weit abstehend, $a a=2 a b$ und $d d=3-4$ mal $c d .^{1}$

Fundort: Mittlere Sandalp.

A. veneta var. hortensis Mich.

Borsten abstehend. $a b=c d ; b c=2 a b ; d d=6 c d ; a a=$ $3 a b$.

Fundorte: Inner Ferrera (Avers).

4. A. alpina Rosa.

(Rosa, I Lumbricidi del Piemonte; Torino 1884.)

Länge ca. $40 \mathrm{~mm}$. Segmente 130. Prostomium $2 / 3$, mit Querfurche. Borsten abstehend: $a b=b c=c d$; $d d=3 c d$; $a a=2 a b$.

Clitellum 27., 28-33., 34.

Fundort: Auf der Frutt, 2000 m., von Herrn Prof. Dr STOLL gefunden.

\section{A. subrubicunda Eisen.}

(Eisen, Om Shandinaviens Lumbricider, Ofversigt K. Vetensk. Akad. Förhandl. 30, 1873.)

Farbe dunkel braunrot, Gürtel hellrot.

${ }^{1}$ Mit $a, b, c, d$ sind die von der ventralen Mediane aus aufeinanderfolgenden Borsten eines Segmentes bezeichnet. 
Länge $50 \mathrm{~mm}$. Durchmesser $4 \mathrm{~mm}$. Segmente 90-115. Borsten abstehend. Prostomium ${ }^{2} / 3$. Fortsatz breit. Clitellum 26., 27-31. Tub. pub. 28., 29., 30. oder 29., 30.

Fundorte: In Oerlikon, auf Hurden, auf der Frutt (Herr Prof. Dr STOLL), im Tierfehd (Glarus), auf dem Pragel,im Avers (am Weisshorn) bis über $2600 \mathrm{~m}$. Höhe in den Polsterrasen von Silene und Saxifraga.

\section{A. constricta Rosa.}

(Rosa, I Lumbricidi del Piemonte 1884.)

Farbe rotbraun. Länge ca. $30 \mathrm{~mm}$., Durchmesser $3 \mathrm{~mm}$. Segmentzahl 90-100. Prostomium $\% / 3$, breit. Borsten abstehend, die dorsalen etwas mehr als die ventralen. Gürtel 26-31. Tub. pub. fehlend. Erster Rückenporus in 5-6. Ohne Spermatheken.

Fundorte: Hurden, Weiningen an der Limmat, an der Glatt.

7. A. octoedra Sav.

(Savigny, l. c.)

Farbe dunkel braunrot, Gürtel lederfarbig, fahl bis gelb. Länge 50-60 mm. Durchmesser $3 \mathrm{~mm}$. Segmentzahl 80-95. Prostomium ${ }^{2} / 3$. Borsten gleich weit von einander abstehend. Gürtel 28., 29-33. Tub. pub. 30., 31-33. O Geschlechtsöffnung 15., Papille auch auf 16. ausgedehnt. Erster Rückenporus in 5-6.

Fundorte: Wiese bei Cresta, Pragelpass (unter Rinde), Hintersandalp und Obersandalp (2100 m.), Tierfehd (Glarus).

8. A. caliginosa Sav. var. trapezoides Rosa. (Savigny, l. c.)

Farbe blassrot, graublau oder braun, Gürtel gelblich oder dunkel. Länge 130-150 mm. Durchmesser 5-7. Segmentzahl 185-275. Prostomium $1 / 3$. Borsten engpaarig. Gürtel 27., 2834. Tub. pub. 31-33. o' Oeffnung 15., lange Querspalte; 14. und 16. ebenfalls angeschwollen. 1 Rückenporus in 9-10. Spermatheken in 9-10. und 10-11. sich öffnend. 
Fundorte: Ueberall in der Umgebung von Zürich; Hurden ; auf dem Albis; Hittnau; Cresta (Avers); St. Gallen (Herr D ${ }^{r}$ Hescheler).

A. caliginosa var. turgida Rosa.

Tub. pub. 31. und 33. Geringere Grösse und Segmentzahl als vorig.

Fundorte: Letzibach (Zürich), Hittnau, Tierfehd (Glarus).

9. A. terrestris Savigny.

(Savigny, l. c.)

Farbe braunrot; Gürtel braun. Länge $140 \mathrm{~mm}$. Durchmesser $5 \mathrm{~mm}$. Segmentzahl 190. Prostomium $1 / 3$; von dessen hintern Ecken aus gehen je 2 Furchen schräg nach aussen. Borsten eng paarig. Gürtel 28-35. Tub. pub. 32-34. Die ventralen Borsten stehen hier auf kleinen Papillen. Segmente 9-11 ventral angeschwollen.

Fundorte: Ueberall in der Umgebung von Zürich.

\section{A. chlorotica Sav.}

(Savigny, l. c.)

Farbe fahl, grünlich; Gürtel schmutzig gelblich. Länge ca. $70 \mathrm{~mm}$. Durchmesser $4 \mathrm{~mm}$. Segmentzahl 110. Prostomium $1 / 2$, breit. Borsten eng paarig. Gürtel 29-36., 37. Tub. pub. 31., 33., 35.; Papillen mit mittlerer Vertiefung.

$\sigma^{x}$ Geschlechtsöffnung deutlich, Papille auf die benachbarten Segmente sich erstreckend. Erster Rückenporus in 4-5.

Fundorte: Ueberall in der Umgebung Zürichs, namentlich auch im Uferschiamm der Limmat, an Bächen.

\section{A. cyanea Savigny var. profuga Rosa.}

Farbe aschblau, Gürtel gelblich. Länge 100-120 mm. Durchmesser $6 \mathrm{~mm}$. Segmentzahl 155. Prostomium $1 / 2$, Borsten abstehend. Gürtel 30-35. Tub. pub. 31-34. Papille der $\sigma^{\Upsilon}$ Geschlechtsöffnung auf 14 . und 16. sich erstreckend. 
DIE OLIGOCHETEN VON ZURICH.

Fundorte: Zürichberg, Käferberg, Hurden, Wädensweil, nicht gerade häufig.

A. cyanea var. studiosa Mich.

Grösser als vor. Clitellum 29-34. Tub. pub. 30-33. 1 Rückenporus in 11-12.

Fundorte: In der Limmat (im Wasser unter einem Stein), an einem Wassergraben im fallend. Brunnenhof (Zürich).

\section{A. hermanni Michaelsen.}

(Michaelsen, Die Lumbriciden Deutschlands. Jahrbuch der Hamburg. wissensch. Anstalten 1890.)

Länge 50-70. Durchmesser 1,5-2 mm. Segmentzahl 140 . Prostomium ${ }^{2} / 3$. Borsten eng paarig, in den vordern Segmenten gross. Gürtel fehlend (?). Tub. pub. 29., 30. O Geschlechtsöffnung deutlich, Papille auch auf 14. und 16. sich erstreckend.

Fundorte: An den Ufern der Bäche, der Limmat und Glatt, des Zürichsees; überall recht häufig.

\section{A. limicola Michaelsen. (Michaelsen, I. c.)}

Farbe blass. Länge $80 \mathrm{~mm}$. Durchmesser $5 \mathrm{~mm}$. Segmentzahl ca. 130. Prostomium $1 / 2$. Borsten eng paarig. Gürtel 29-35. Tub. pub. 33., 34. Borsten eng paarig. Erster Rückenporus in 4-5.

Fundort: Bei der Einmündung der Reppisch in die Limmat (1 Exemplar) im Kies.

\section{Genus : Lumbricus Eisen.}

Das Prostomium teilt das Peristomium vollständig. Borsten eng paarig, auf dem Clitellum länger. Mit mittlerer Samenblase in 10. und 11., in welche 3 Paar Samensäcke sich ergiessen. 2 Paar Spermatheken in 9. und 10. 


\section{L. rubellus Hoffmeister.}

(Hoffmeister, Beitrag zur Kenntnis deutscher Landanneliden. Archiv

f. Naturgesch. 1843.)

Farbe rotbraun, iridisirend, selten dunkeliraun; Clitellum heller. Länge 70-90 mm. Segmentzahl 80-115. Fortsatz des Prostoms mit oder ohne Querfurche. Clitellum 27-32. Tub. pub. 28-31. O Geschlechtsöffinung wenig deutlich. 1 Rückenporus in 7-8.

Fundorte: Ueberall um Zürich; Hurden; Tierfehd (Glarus); Thusis; im Avers bis 2500 m. Höhe.

\section{L. castaneus Sav.}

(Savigny, l. c.)

Farbe braunviolett oder rotbraun; Clitellum hellrot; stark rridisirend. Länge 40-50 mm. Clitellum 28-33. Tub. pub. 2932. $\sigma^{\Upsilon}$ Geschlechtsöffnung sehr undeutlich.

Fundorte: Hurden, unter angeschwemmtem Schilf; Limmat, im Schlamm; Oerlikon, am Bachrand; Zürich IV, im Kies von Gartenwegen; Frutt (Herr Prof. Dr STOLL).

\section{L. melibcus Rosa.}

(Rosa, I Lumbricidi del Piemonte 1884.)

Farbe rotbraun. Länge $60 \mathrm{~mm}$. Durchmesser $5 \mathrm{~mm}$. Clitellum 29-33. Tub. pub. 30-33. O' Geschlechtsöffnung deutlich.

Fundort: Affoltern bei Höngg, in fast ausgetrocknetem Bachbett.

\section{L. herculeus Savigny.}

(Savigny, l. c.)

Farbe dunkel rotbraun; Clitellum lederfarbig. Länge $170 \mathrm{~mm}$. Durchmesser 9-10 mm. Segmente 155. Prostomium ohne oder mit 1-2 Querfurchen. Clitellum 32-37., 38. Tub. pub. 33-36. Männliche Geschlechtsöffnung deutlich.

Fundorte: Ueberall in der Umgebung von Zürich, nicht selten. 


\section{B. Bestimmungstabelle.}

\section{GATTUNGEN}

Prostomium in das Mundsegment hinein verlängert; Regenwürmer...... 1

Prostomium nicht in das Mundse sment verlängert................ 2

1. Borslen eng paarig ; Prostom $1 / 1 \ldots . . . \ldots . . .$. XXIII. Lumbricus. oder abstehend; wenn jene engpaarig, Prostom

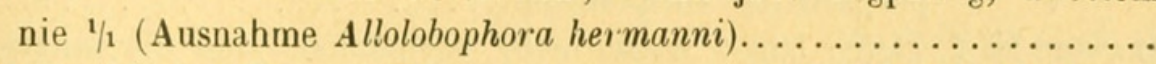

2. $\sigma^{7}$ Geschlechtsöffnung in $15 \ldots \ldots \ldots \ldots \ldots \ldots$ XXII. Allolobophora. $\sigma^{\pi} \quad$ in $13 \ldots \ldots \ldots \ldots \ldots \ldots \ldots \ldots \ldots \ldots \ldots \ldots \ldots$ XXI. Allurus.

3. Borsten fehlend; nur Drüsen vorhanden........... XX. Anachæta.

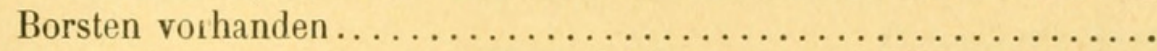

4. Borsten nur veníral, doppelhakig; zu 4-10 per Bündel. XV.Chætogaster. Borsien dorsal und ventral (wenn nur ventral deutlich, nicht doppel-

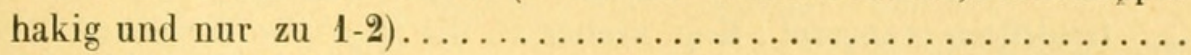

5. Ausschliesslich Haarborsten...................... Aeolosoma.

Neben Haar- auch andere Borsten, oder jene Form ganz fehlend....

6. Dorsal nur Haar-, oder Haar- und Haken- (auch Kamm-) borsten.... Ausschliesslich Haken- oder einfache Borsten.............. 16

7. Rückenborsten in 5 oder 6 beginnend.................... 8 , schon in 2 beginnend ..................... 9

8. Mit Kiemenanhängen am Hinterende............. XIV. Dero. Ohne Kiemenanhänge am Hinterende............... Nais.

9. Prostom in einen Rüssel verlängert............... PI. Pristina.

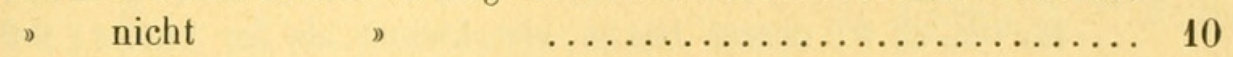

10. Dorsal nur Haarborsten.......................... 11

» neben Haar- auch andere Borstenformen............... 12

11. Rückenborsten bis 2 mal länger als der Durchmesser des Tieres; ohne Sinnespapillen...................... XIII. Macrochæta. Rückenborsten kürzer als der Durchmesser des Tieres ; mit Sinnespapillen........................... Embolocephalus.

12. Kammborsten undeutlich gezähnt............... VI. Tubifex. ه deutlich, fächerförmig............VIII. Psammoryctes.

16. Dorsal nur Hakenborsten; die der vordersten Segmente schlanker als in den folgenden..................... XII. Homochæta. Alle dorsalen Borsten gleichartig, hakig oder einfach.............

17. Borsten dorsal zu mehr als 2 per Bündel, doppelhakig; Haken fast gleich gross........................... Limnodrilus. Borsten dorsal nur zu 2, einzeln oder fehlend; wenn mehr vor-

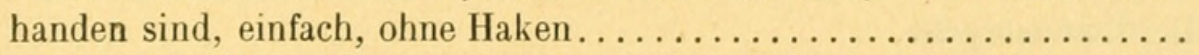

18. Blinde Gefässäste an den Perivisceralschlingen vorhanden.......... Blinde Gefässäste fehlend............................

Rev. Suisse de Zool., T. III. 1896. 
K. BRETSCHER.

19. In jedem Segment nur eine Schleife mit blinden Aesten. III. Lumbriculus.

2 Schleifen in jedem Segment mit blinden Aesten... V. Claparedilla.

20. Penis nach aussen vortretend, nicht retraktil.........IV. Stylodrilus.

Penis retraktil, gewöhnlich nicht nach aussen vortretend.........

21. Borsten zu 2, ohne Haken; Körper sehr lang, über 200 Segmente.

II. Phreoryctes.

Borsten zu mehr als 2; Körper kurz, weniger als 100 Segmente

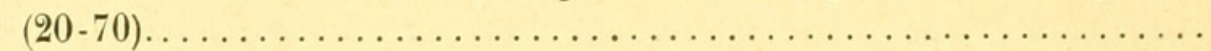

22. Blut rot; Borsten einfach, zu 4-7........... PVI. Pachydrilus.

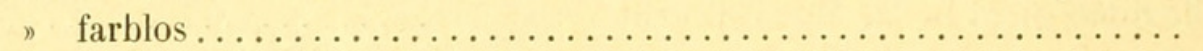

23. Rückengefäss anteclitellial entspringend........... XIX. Henlea.

postclitellial entspringend.........................
,en zu 2, gleich lang; wenn mehr vorhanden, sind die inneren

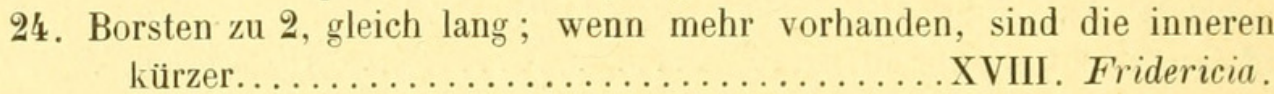
Borsten zu 3 oder mehr; gleich lang..........XVII. Enchytraeus.

\section{ARTEN}

I. Aeolosoma.

Oeldrüsen orangerot; Kopf breiter als die folgenden Segmente.

Ae. hemprichii Ehrbg.

Oeldrüsen farblos, Kopf nicht breiter als die folgenden Segmente.

II. Phreoryctes.

Ae. fiedleri nov. spec.

Ventrale Borsten länger als die dorsalen; gegen 500 Segmente.

$\mathrm{Ph}$. menkeanus Hoffm.

Dorsale Borsten länger als die ventralen ; 200-300 Segmente.

Ph. filiformis Clap.

III. Lumbriculus.

Borsten zu 2; oberer Haken viel kleiner als der untere; 6-8 blinde Gefässäste in den hintern Segmenten....L. variegatus 0. F. Müller.

IV. Claparedilla.

Prostomium nicht länger als das Mundsegment.

V. Stylodrilus.

C. meridionalis Vejdovsky.

Penis fast so lang als der Durchmesser des Tieres.

IV. Tubifex.

St. gabretæ Vejdovsky.

Dorsale Borsten haar-, kamm- und hakenförmig.

VII. Limnodrilus.

T. rivulorum Lamarck.

Chitinöser Penis 8-10 mal länger als breit....L. claparedianus Ratzel.

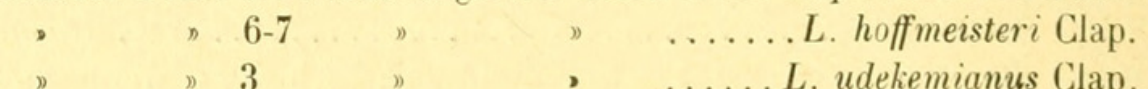

VIII. Psammoryctes.

In den vordern 11 Segmenten neben Haar- auch Fächerborsten.

$P$. barbatus Grube. 
IX. Embolocephalus.

Rückenborsten zu 2-4................... velutinus Grube. $6-9 \ldots \ldots \ldots \ldots \ldots \ldots$. . plicatus Randolph. X. Nais.

Mit langem Rüssel....................... lacustris Linné. Ohne Rüssel............................ 1

1. Mit Schlammhülle........................... 2 Ohne Schlammbülle......................... 3

2. Rückenborsten alle kurz; vorn 3-4 dunkle Pigmentstreifen.

N. serpentina 0 . F. Müller. Rückenborsten in 6. sehr lang, ohne dorsale Pigmentstreifen.

$N$. appendiculata d'Udeken.

3. Dorsal nur Haarborsten................. barbata 0. F. Müller.

" Haar- und Hakenborsten........... elinguis 0. F. Müller.

XI. Pristina.

Dorsale Borsten in 3. viel länger als die übrigen

P. longiseta Ehrenberg.

XII. Homochrta.

Alle Borsten doppelhakig; Rückenborsten schon in 2.

H. naidina nov. spec.

XIII. Macrochæta.

Dorsale Borsten in 6 . beginnend, nicht gesägt, alle lang.

XIV. Dero.

M. intermedia nov. spec.

Körper hinten (ausser den Kiemenzapfen) in 2 lange Tastfortsätze endend.

D. furcata $0 \mathrm{ken}$.

Körper ohne solche Fortsätze..................... 1

1.4 Kiemenanhänge .................... perrieri Bousf. 6

D. digitata 0 . F. Müller.

XV. Chaetogaster.

Oesophagus sehr verkürzt; parasitisch lebend. . Ch. linmnaei von Bær. Oesophagus fast so lang als der Pharynx............... 1

1. Blutgefässe nicht in den Pharynx verlaufend.

Ch. cristallinus Vejdovsky.

Blutgefässe in den Pharynx reichend................. 2

2. Blutgefässe mit dichtem Zellbelag; Kopflappen spitzig.

Ch. diastrophus Gruithuisen.

Blutgefässe mit Zellen spärlich besetzt................ 3

3. $15 \mathrm{~mm}$. lang; Darm meist mit kleinen Krebsen, Schnecken, Rotatorien gefüllt................. diaphanus Gruithuisen.

$2 \mathrm{~mm}$. lang; Darm mit Algen und Diatomeen.Ch. langi nov. spec.

XVI. Pachydrilus.

Hautdrüsen farblos; Borsten ventral zu 7-10; 15 -20 mm. lang.

$P$. pagenstecheri Ratzel.

Hautdrüsen im Kopf- und Analsegment orangerot; 5 mm. lang.

P. maculata nov. spec. 
XVII. Enchytræus.

Anteseptale ohne Kanal, Samentasche mit basalen Drüsen.

E. buchholzii Vejdovsky.

XVIII. Fridericia.

4 Seitentaschen der Spermatheken, nicht gestielt..F. galba Hoffmeister.

2 Seitentaschen der Spermatheken ................... 1

1. Seitentaschen gross; Anteseptale braun......F. leydigii Vejdovsky.

Seitentaschen gross; Anteseptale hell.....F. perrieri Vejdovsky.

Seitentaschen klein, deutlich gestielt wie die Endblase.

$F$. helvetica nov. spec.

XIX. Henlea.

2 Oesophagealdrüsen.................... leptodera Vejdovsky. 4 H. ventriculosus d'Udeken.

XX. Anachæta.

2 Reihen Borstendrüsen................ bohemica Vejdovsky.

XXI. Allurus.

Clitellum. 22., 23-27.

Tub. pub.

23-25., 26. A. tetraëdrus Sav.

XXII. Allolobophora.

I. Borsten abstehend.

Clitellum.

26., 27-32., 33 .

27., 28-33., 34 .

26 . $27-31$.

26-31.

28., 29-33.

Tub. pub.

$$
\begin{aligned}
& \text { 30., 31. A. veneta Rosa. } \\
& \text { 30-32. A. alpina Rosa. } \\
& \text { 28., 29-30. A. subrubicunda Rosa. } \\
& \text { fehlt A. constricta Rosa. } \\
& \text { 30., 31-33. A. octö̈dra Sav. }
\end{aligned}
$$

II. Borsten eng paarig.

Clitellum.

24., $23 ., 26-32$.

2อ., $26 \cdot 32$.

27., 28-34.

$27 ., 28-34$.

28-3วั.

29-36., 37 .

29., 30-34., 35ั.

22-32.

29-35.

XXIII. Lumbricus .

Clitellum.

27-32.

28-33.

29-33.

32-37.
Tub. pub.
28-30., 31. A. fœetida Sav.
29-31. A. rosea Sav.
31-33. A. caliginosa Sav.
31-33. A. caliginosa var. turgida.
32-34. A terrestris Sav.
31., 33., 35ั. A. chlorotica Sav.
30., 31-33., 34. A. cyanea Sav.
29., 30. A. hermanni Michælsen.
33., 34. A. limicola Michælsen.

Tub. pub.

28-31. L. rubellus Hoffm.

29-32. L. castaneus Sav.

30-33. L. melibœus Rosa.

33-36. L. herculeus Sav. 


\section{Biologisches.}

Hinsichtlich des Eintritts der Sexualität bei einigen Oligochæten seien nur folgende Beobachtungen erwähnt. Lumbriculus variegatus legt die Geschlechtsorgane erst im November an. Bei Chaetogaster diaphanus und diastrophus zeigten einzelne Exemplare dieselben Anfang und Ende Oktober, ohne dass eine deutliche Gürtelbildung zu Tage getreten wäre, die bei Ch. langi so ausgesprochen war. Nais lacustris tritt Ende September allgemein in den Zustand der Geschlechtsreife, während daneben wenigstens durch einige Zeit die ungeschlechtliche Vermehrung sich erhält. Nais appendiculata entwickelte die Sexualorgane Ende Oktober. Namentlich interessant ist aber in dieser Hinsicht das Verhalten von Nais elinguis und Pristina longiseta. Erstere Art beobachtete ich mehrmals, schon im Mai, dann im Juni u. August in geschlechtlichem Zustande und zwar im Wasserglase, wie in Wassergräben, die am Austrocknen waren. Gleichzeitig fanden sich in günstigen Existenzbedingungen nirgends Objekte in diesem Stadium! Pristina longiseta traf ich zweimal so; doch nimmt bei diesem Tiere die Entwicklung der Sexualorgane anscheinend mehr Zeit in Anspruch als bei $N$. elinguis. Es wird also hier der Eintritt der Geschlechtsreife durch äussere Verhältnisse bedingt. Dies beweisen übrigens auch die Befunde von Vejdovsky und Timm.

Erwähnenswert ist auch der Umstand, dass ich $P$. longiseta niemals in Teilung, mit Sprosszonen, begriffen fand, ein Verhalten, das alle übrigen Naidomorphen durchweg aufweisen.

Sinnespapillen besitzen Nais appendiculata, Embolocephalus velutinus und plicatus, ebenso aber auch $N$. serpentina, alles Formen, die normalerweise in einer dichten Hülle von Schlamm, pflanzl. Abfällen, Diatomeen stecken. Die erwähnten Organe 
dienen mithin offenbar dazu, die durch jenes Kleid verminderte Sensibilität des Körpers wieder herzustellen. Es hält jedoch für gewöhnlich recht schwer, die Sinneshügel von $N$. serpentina aufzufinden, bei dem sie in einer Ringzone um jedes Segment angeordnet liegen. Dieses Vorkommniss bei $N$. serpentina scheint wenig beachtet worden zu sein.

Von Schmarotzern fand sich in einem Chaetogaster diaphanus ein Fadenwurm im Pharyngealabschnitt; Pachydrilus pagenstecheri zeigte nicht selten Urocystis saenuris; einige wenige Tubifex rivulorum enthielten Insassen, die den von CLAPARÈDE erwähnten Opalinen entsprechen dürften. Ein Psammoryctes war mit einem Tiere in der Leibeshöhle behaftet, das Herr Prof. ZschoKKE in Basel, dem ich das Objekt zusandte, als ein Jugendstadium eines Caryophyllaeus diagnostizirte. Ferner enthielten Nais lacustris vom Zürichsee (Bucht am Zürichhorn) im Herbst oft Bewohner im Darme, seltener in der Leibeshöhle, die gewöhnlich in Mehrzahl vorhanden waren, und über deren $\mathrm{Zu}$ gehörigkeit ich nichts in Erfahrung bringen konnte. Es dürfte sich aber wohl um Gregarinen handeln. Ein Lumbricus herculeus zeigte unter der Cuticula einen Enterion(HoffMeister, Die bis jetzt bekannten Arten der Regenwïrmer, 1845).

Nicht ohne Interesse sind die Verbreitungsverhältnisse der Lumbriciden. $\mathrm{Zu}$ den gewöhnlichen Erscheinungen gehören Lumbricus rubellus, herculeus; Allolobophora hermanni, chlorotica, terrestris, caliginosa, rosea; Allurus tetraëdrus. Lumbricus castaneus fand sich in einem Garten in den Kieswegen zahlreich, während er in den Beeten fehlte; recht vereinzelt tritt in der Umgebung von Zürich L. melibceus auf. Allolobophora rosea scheint unter verwesendem Laub der Wälder mit Vorliebe sich anzusiedeln; $A$. fretida dagegen bewohnt in Menge Dünger- und Komposthaufen, wo er gerade die Schichten bevorzugt, welchen in Folge der Zersetzung des aufgehäuften Materials eine bedeutende Wärme eigen ist. Dahin werden auch die Cocons abgelegt. 
In den Alpenweiden traf ich in der Erde, welche allerdings hier meist in sehr dünner Schicht die Felsen bedeckt, höchst selten Würmer; sie sind ein geradezu ausnahmsweises Vorkommnis; vereinzelt erscheinen sie in den Lagerstellen und unter den Exkrementen der Weidetiere sammeln sie sich geradezu an, so dass oft bis 10 und noch mehr Exemplare unter einem einzigen Haufen zu treffen sind. Meine Funde gehörten ausschliesslich Allolobophora subrubicunda an. Da sie zu ihrer Existenz eines gewissen Masses von Feuchtigkeit bedürfen, so sind die Tiere hier auf ein Wanderleben angewiesen. Die vertrockneten Exkremente können ihnen nicht mehr als Obdach und Nährboden zusagen; sie müssen passendere Existenzbedingungen aufsuchen. Dasselbe lässt sich behaupten von den vereinzelten A. subrubicunda, welche die Silene- und Saxifraga-Polster am Weisshorn im Avers, in $2640 \mathrm{~m}$. Höhe, also hart an der Grenze des ewigen Schnees, besiedelten. Der Fortpflanzungstrieb zwingt sie hier zur Wanderschaft, auf der ihnen die Polsterrasen ohne Zweifel genügend Nahrung und einen guten Unterstand gewähren.

Welchen Anteil die Lumbriciden an der Bodenfauna nehmen, ergiebt sich aus einzelnen vorgenommenen Ausgrabungen. Sie ergaben in einer Wiese in der Nähe von Zürich 70-230, in einem Acker 140-260, im Wald ca. 90 Stück auf 1 m. ${ }^{2}$, während die Ausbeute in einer Wiese bei Cresta, $1950 \mathrm{~m}$., wo der Humus in $15 \mathrm{~cm}$. Tiefe die felsige Unterlage deckt $u$. der Untergrund vollständig fehlt, auf die gleiche Fläche 1660 Stück ergab, zu denen noch eine sehr beträchtliche Zahl,wohl nicht weniger,Enchytræen sich gesellten. Der letztere Fund bestand aus Allolobophora rosea, cyanea, octoëdra und Lumbricus rubellus. Die ,,grossen Arten“, die Hensen in seinen bedeutenden Arbeiten über den Einfluss der Regenwürmer auf die Pflanzenwelt erwähnt und zu denen wohl L. herculeus, Allolobophora terrestris und caliginosa zu rechnen sind, fehlen also hier, während sie allerdings in der 
Ebene meist die grosse Mehrzahl ausmachen. Es wird durch erneute und zahlreichere Nachgrabungen, die immer sehr zeitraubend sind, nachzuweisen sein, ob dieses Verbreitungsverhältnis allgemein ist. Dann müsste wohl angenommen werden, dass für die letztgenannten Arten das Vorhandensein eines Untergrundes zum mindesten eine wichtige Existenzbedingung ist.

Weitere Angaben über die Lumbriciden-Fauna der Schweiz machen HagenBach im Naturwissenschaftl. Anzeiger d. allg. schweiz. Gesellsch. f. d. gesamte Naturkunde. Sein Lumbricus leptozonicus ist sehr wahrscheinlich identisch mit Allolobophora fretida. Michaelsen erwähnt im Archiv für Naturgeschichte, 1892, I : Die Terricolen der Berliner zoologischen Sammlung II:

Lumbricus rubellus vom Weissbad (Appenzell) und Oberengadin.

All 3!) bophora ocioëdra vom Berninapass.

" subrubicunda Eisen vom Berninapass und

» cyanea var. profuga vom Weissbad. 


\section{$2 \mathrm{BHL}$ Biodiversity Heritage Library}

Bretscher, Konrad. 1896. "Die Oligochoeten von Zürich in systematischer und biologischer Hinsicht." Revue suisse de zoologie 3(4), 499-532. https://doi.org/10.5962/bhl.part.117941.

View This Item Online: https://www.biodiversitylibrary.org/item/38140

DOI: https://doi.org/10.5962/bhl.part.117941

Permalink: https://www.biodiversitylibrary.org/partpdf/117941

\section{Holding Institution}

MBLWHOI Library

\section{Sponsored by}

MBLWHOI Library

\section{Copyright \& Reuse}

Copyright Status: NOT_IN_COPYRIGHT

This document was created from content at the Biodiversity Heritage Library, the world's largest open access digital library for biodiversity literature and archives. Visit BHL at https://www.biodiversitylibrary.org. 